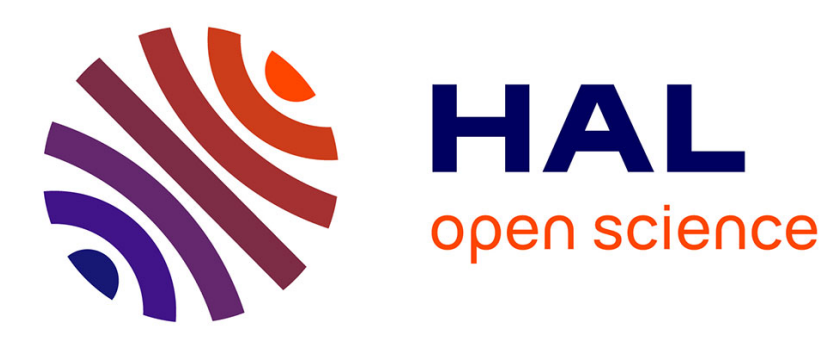

\title{
Influence d'un déficit hydrique sur trois variétés de soja : effet sur la protéogenèse des graines
} Jean Calmés, Guy Viala., Noël Gelfi, Robert Blanchet, J. Laurent

\section{To cite this version:}

Jean Calmés, Guy Viala., Noël Gelfi, Robert Blanchet, J. Laurent. Influence d'un déficit hydrique sur trois variétés de soja: effet sur la protéogenèse des graines. Agronomie, 1985, 5 (2), pp.169-176. hal-00884746

\section{HAL Id: hal-00884746 \\ https://hal.science/hal-00884746}

Submitted on 1 Jan 1985

HAL is a multi-disciplinary open access archive for the deposit and dissemination of scientific research documents, whether they are published or not. The documents may come from teaching and research institutions in France or abroad, or from public or private research centers.
L'archive ouverte pluridisciplinaire HAL, est destinée au dépôt et à la diffusion de documents scientifiques de niveau recherche, publiés ou non, émanant des établissements d'enseignement et de recherche français ou étrangers, des laboratoires publics ou privés. 


\title{
Influence d'un déficit hydrique sur trois variétés de soja : effet sur la protéogenèse des graines
}

\author{
Jean CALMÉS, Guy VIALA, Noël GELFI $\left(^{*}\right)$ \& Robert BLANCHET $(*)$ \\ avec la collaboration technique de J. LAURENT \\ Centre de Physiologie végétale, Laboratoire associé au C.N.R.S. $n^{\circ} 241$, Université Paul Sabatier, 118, route \\ de Narbonne, F 31062 Toulouse Cedex \\ (*) I.N.R.A., Station d'Agronomie, Centre de recherches de Toulouse, B.P. 12, F 31320 Castanet-Tolosan
}

\begin{abstract}
La tolérance au déficit hydrique de 2 variétés de soja (Glycine max (L.) Merr.) d'origine chinoise, " Jiangedou " et "Quingmoshidou 533 » ("Q.533») est comparée à celle de la variété “ Kingsoy » couramment cultivée en France.

Des parcelles dont les plantes sont soumises à une contrainte hydrique sévère depuis le début de la floraison jusqu'à la fin de la formation des gousses (1 ${ }^{\text {er }}$ juillet-15 août) sont comparées à des parcelles témoins placées dans des conditions d'évapotranspiration maximale. Les répercussions de ce traitement sur le développement végétatif et la fructification, les échanges gazeux et le métabolisme carboné et azoté sont étudiées.

Durant la période de déficit hydrique, la réduction des échanges gazeux foliaires et de l'alimentation azotée entraîne une croissance limitée et une fructification déficitaire. Le marquage, après incorporation de ${ }^{14} \mathrm{CO}_{2}$, des composés insolubles non protéiques est plus important dans les feuilles en déficit hydrique que dans les témoins : ceci témoigne d'une synthèse protéique moins active.

Dix jours après avoir supprimé la contrainte hydrique, la synthèse protéique reste plus faible par rapport aux témoins, ceci est particulièrement net pour «Q.533 ». Par ailleurs, le comportement de cette variété, dont le développement végétatif reprend au point d'atteindre celui du témoin, diffère de celui de "Jiangedou » et de « Kingsoy ».

Le ralentissement du métabolisme carboné et surtout azoté, provoqué par le déficit hydrique, se traduit, à maturité, par une réduction de la masse des graines récoltées, plus importante pour «Q.533 ». Pour les trois variétés la proportion des protéines dans les graines n'a pas été modifiée par le déficit hydrique étudié.
\end{abstract}

Mots clés additionnels : Echanges gazeux, métabolisme carboné, acides aminés, protéines, productivité.

Influence of water stress on three soybean varieties : effect on seed proteogenesis.

\begin{abstract}
The drought tolerance of 2 chinese soybean varieties (Glycine $\max$ (L.) Merr.) 'Jiangedou' and 'Quingmoshidou 533' ('Q.533'), was compared with that of 'Kingsoy', currently cropped in France. Plots were water-stressed from flowering until pod formation (August 15) and compared with standard plots in maximum evapotranspiration conditions. The effects of this treatment were studied on vegetative growth and fruiting, gaseous exchange, carbon and nitrogen metabolism. During water stress, reduction of gaseous exchange and nitrogen adsorption led to reduced growth and weak fruiting. After ${ }^{14} \mathrm{CO}_{2}$ incorporation, labelling of nonprotein insoluble compounds was greater in water-stressed than standard leaves, showing less protein synthesis. Ten days after the end of water stress, protein synthesis remained lower by comparison with standard, particularly for ' $Q .533$ '. In fact, this variety's behaviour was generally very different from that of 'Jiangedou' and 'Kingsoy' : vegetative growth increased again to the control level. Water stress decreased carbon and especially nitrogen metabolism and led to a reduction of seed weight at maturity particularly for ' $Q .533$ '. The seed protein level of the three varieties was not modified by the water stress studied.
\end{abstract}

Additional key words : Gaseous exchange, carbon metabolism, amino acids, proteins, productivity.

\section{INTRODUCTION}

L'effet d'un déficit hydrique sur la fructification des plantes cultivées a fait l'objet de nombreux travaux comme en témoignent les revues générales établies par HSIAO (1973), PALEG \& ASPINALL (1981),
BRADFORD \& HSIAO (1982). La tolérance du soja (Glycine max (L.) Merr.) à la sécheresse comporte à la fois des aspects anatomiques et morphologiques (VIDAL et al., 1981 ; POGNONEC, 1983) et des aspects physiologiques (PLANCHON \& VIGNES, 1978 ; FINN \& BRUN, 1980 ; BOUNIOLS et al., 1982 ; BALDOCCHI et 
TABLEAU 1

Données climatiques et caractéristiques de l'alimentation hydrique. Les plantes témoins sont en évapotranspiration maximale (ETM); les plantes traitées sont en évapotranspiration réelle (ETR).

Climatic characters and water consumption. Standard plants in maximum evapotranspiration (ETM). Treated plants in real evapotranspiration (ETR).

\begin{tabular}{|c|c|c|c|c|c|c|c|}
\hline & mai & juin & juil. & $\begin{array}{l}\text { août } \\
(1-15)\end{array}$ & $\begin{array}{c}\text { août } \\
(16-31)\end{array}$ & sept. & oct. \\
\hline Températures moyennes $\left({ }^{\circ} \mathrm{C}\right)$ & 13,4 & 19,3 & 29,6 & 27,1 & 25,8 & 18,9 & 14,3 \\
\hline Pluies (mm) & 51 & 32 & 9 & 2 & 165 & 16 & 28 \\
\hline \multicolumn{8}{|l|}{ Eau consommée $(\mathrm{mm})$} \\
\hline en ETM : témoin & 45 & 135 & 225 & 140 & 95 & 120 & 30 \\
\hline Rapport ETR/ETM & 1 & 0,8 & 0,6 & 0,4 & 1 & 1 & 1 \\
\hline
\end{tabular}

al., 1983) qu'il n'est pas toujours aisé de relier à la production de graines (SAMMONS et al., 1978, 1979 ; MAERTENS \& BLANCHET, 1981). De plus, les effets du manque d'eau diffèrent suivant l'âge physiologique de la plante (HUCK et al., 1983).

Dans le Sud-Ouest de la France, l'été est généralement marqué par une période plus ou moins longue de sécheresse intense qui se situe le plus souvent à la fin du mois de juillet et au début du mois d'août, au moment où le soja fleurit et développe ses gousses. Cette époque correspond, dans le cas de variétés de type indéterminé, à la fin du développement végétatif pour le sommet de la plante et au début de la fructification pour la base : ceci leur confère une souplesse généralement reconnue à l'égard de la contrainte hydrique.

Ce travail se propose d'étudier les modifications du métabolisme carboné et azoté induites par le déficit hydrique, d'une part, pendant la période de sécheresse et, d'autre part, lorsque les plantes sont à nouveau alimentées en eau. Les répercussions sur les organes végétatifs et reproducteurs sont également examinées et ceci pour 3 variétés différentes qui ont paru présenter un intérêt particulier lors d'expérimentations antérieures.

\section{MÉTHODES EXPÉRIMENTALES}

\section{A. Matériel végétal}

Trois cultivars de soja, de type indéterminé, sont étudiés : « Kingsoy », originaire des U.S.A., groupe de précocité II, est le plus cultivé en France; sa production et sa résistance à la sécheresse sont bonnes, bien que sa consommation d'eau soit assez élevée. « Jiangedou » (1), originaire de Mandchourie, dont la précocité est voisine de " Kingsoy ", présente une productivité et une consommation d'eau un peu plus faibles. "Quingmoshidou 533 » ( Q Q.533»), originaire de Mandchourie, un peu plus tardif que "Kingsoy ", est très vigoureux avec des tiges tendant à former des lianes; il présente une régulation stomatique importante lors d'une demande évaporative élevée (BLAN-

(1) Les graines de " Jiangedou " et " Quingmoshidou 533 " nous ont été fournies par des agronomes chinois dans le cadre d'un accord de coopération entre la France et la République Populaire de Chine.
CHET et al., 1982) : sa fructification est très sensible aux contraintes hydriques.

Les 3 variétés sont cultivées au champ, d'une part, avec une irrigation satisfaisant continuellement l'évapotranspiration maximale (témoins ETM), d'autre part, sans arrosage jusqu'au 15 août (plantes traitées ETR). Le tableau 1 précise l'ensemble des données climatiques pour l'année 1983 .

Le sol brun, limono-argileux profond, bien fertilisé en phosphore et potassium, est inoculé avec Rhizobium japonicum et n'a pas reçu d'engrais azotés. Des lots de 10 plantes bien représentatives du peuplement végétal ( 40 plantes. $\left.\mathrm{m}^{-2}\right)$ sont prélevés sur les 2 types de culture aux stades successifs suivants :

- préfloraison (22 juin)

- pleine floraison - début formation des gousses (19 juillet)

- fin formation des gousses (16 août : cette date correspond à la fin floraison pour " Q.533 »)

- remplissage des gousses (5 septembre).

La maturité intervient vers le 15 octobre pour «Kingsoy » et "Jiangedou » et à la fin du mois d'octobre pour «Q.533». A chaque prélèvement, les surfaces foliaires et les masses de matière sèche des tiges, feuilles, gousses et graines sont déterminées, ainsi que leur teneur en azote.

Les échanges gazeux sont mesurés à plusieurs reprises pendant et après la période de déficit hydrique. Des incorporations de ${ }^{14} \mathrm{CO}_{2}$ et de glycolate- $1-{ }^{14} \mathrm{C}$ sont effectuées à l'époque où le manque d'eau est le plus sévère ( 11 août) et $10 \mathrm{j}$ après la fin du déficit hydrique (26 août).

\section{B. Techniques utilisées}

Les incorporations de ${ }^{14} \mathrm{CO}_{2}$ sont effectuées in situ en enfermant la foliole centrale d'une feuille bien ensoleillée durant $30 \mathrm{mn}$ dans une enceinte de $400 \mathrm{~cm}^{3}$ où l'on fait dégager $\mathrm{du}{ }^{14} \mathrm{CO}_{2}$ par action de l'acide lactique sur du bicarbonate de sodium marqué au ${ }^{14} \mathrm{C}$ (radioactivité spécifique : $2050 \mathrm{MBq}$. mmole $^{-1}, \mathrm{CEA}$ ). La technique d'incorporation foliaire du glycolate $-1{ }^{14} \mathrm{C}$ (radioactivité spécifique : $326 \mathrm{MBq} . \mathrm{mmole}^{-1}$, Amersham Radiochemical Centre) a été précédemment décrite (CALMÉS \& VIALA, 1981). Le matériel végétal, prélevé $72 \mathrm{~h}$ après l'incorporation, est conservé au froid, à $-18^{\circ} \mathrm{C}$. Les conditions de fixation, d'extraction, de séparation et d'analyse chromatographique des fractions soluble et 
insoluble ont été exposées par ailleurs (CALMÉs et al., 1977). Les acides aminés sont dosés à l'" amino acid analyser » Beckman, type $119 \mathrm{BL}$.

Les mesures de résistance stomatique et de transpiration effectuées à l'aide du poromètre Li-Cor 1600 sont réalisées sur des feuilles adultes bien ensoleillées, au cours d'après-midi de journées présentant des demandes évaporatives variables suivant les périodes, mais toujours relativement élevées. Les mesures de photosynthèse brute sont effectuées par la méthode de SHIMSHI (1969) qui consiste à exposer $1,5 \mathrm{~cm}^{2}$ de feuille pendant $15 \mathrm{~s}$ à de l'air contenant une quantité connue de $\mathrm{CO}_{2}$ radioactif ( 350 vpm $\mathrm{CO}_{2}$ à $92 \mathrm{kBq}$. mmole $^{-1}$ ) ; l'échantillon est aussitôt prélevé à l'emporte-pièce et, après traitement, la quantité de ${ }^{14} \mathrm{CO}_{2}$ assimilée est dosée au compteur à scintillation.

\section{RÉSULTATS}

\section{A. Croissance, développement et production}

Les figures 1 et 2 indiquent respectivement les évolutions, au cours du cycle de développement, des surfaces foliaires et des masses de matière sèche des organes aériens récoltés. En l'absence de contrainte hydrique, les 3 cultivars développent des feuillages différents, plus abondants chez "Q.533» (indice foliaire de 11 à la fin de la floraison) et chez "Jiangedou" que chez " Kingsoy ». Les productions de masse végétale sèche sont cependant sensiblement identiques jusque peu avant la maturité ; à cette époque, «Q.533 » conserve ses feuilles, tandis qu'elles tombent progressivement chez "Kingsoy » et "Jiangedou ".

Sous contrainte hydrique, l'expansion foliaire est très fortement diminuée, surtout par la taille moyenne des feuilles plutôt que par le nombre de nœuds formés. Les surfaces foliaires sont alors assez voisines chez les 3 cultivars, de même que les productions de matière sèche totale.

C'est à partir de la cessation de la contrainte que "Q.533 " se différencie très fortement des 2 autres variétés : les feuilles de la base restent plus vertes, contrairement à celles des plantes témoins, et les étages supérieurs se développent abondamment, donnant même une nouvelle floraison ; ainsi, chez "Q.533 », la surface foliaire rejoint presque celle des plantes témoins. Il en résulte une forte augmentation de la matière sèche dont l'essentiel demeure toutefois dans les organes végétatifs.

Le tableau 2 indique les productions finales de graines. Les processus de fructification sont très affectés chez les 3 cultivars par la contrainte hydrique, mais de manière un peu différente : diminution du nombre de gousses et du nombre moyen de graines par gousse chez "Kingsoy » et "Jiangedou », diminution plus importante du nombre de gousses chez «Q.533». Pour cette dernière variété, le maximum de déficit hydrique intervient au cours de la floraison tandis que, pour les 2 autres, il se produit à un stade de développement un peu plus avancé. Le grossissement des graines est par contre normal chez les 3 variétés, ce qui n'est pas surprenant car la contrainte hydrique a cessé à ce stade (la taille plus petite des graines de «Q.533 » est un caractère génotypique). Les teneurs en protéines et en huile ne sont pas modifiées.

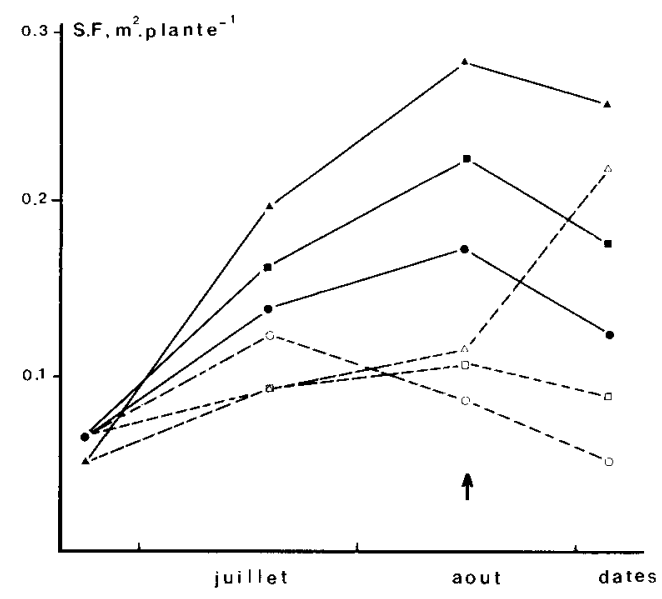

Figure 1

Evolution de la surface foliaire $\left(\mathrm{m}^{2}\right.$. plante $\left.{ }^{-1}\right)$. Parcelles témoins en conditions d'évapotranspiration maximale (Kingsoy $\bullet$, Jiangedou - Q.533 М); parcelles traitées en déficit hydrique jusqu'au 15 août (Kingsoy $\bigcirc$, Jiangedou $\square, Q .533 \triangle$ ); $\uparrow$, fin du déficit hydrique.

Change in leaf area $\left(m^{2}\right.$. plant $\left.{ }^{-1}\right)$. Standard plots in maximum evapotranspiration conditions (Kingsoy $\bullet$, Jiangedou $\mathbf{\square}, Q .533 \mathbf{\Delta}$ ). Treated plots in water stress until August 15 (Kingsoy O, Jiangedou $\square, Q .533 \triangle) ; \uparrow$, end of water stress.

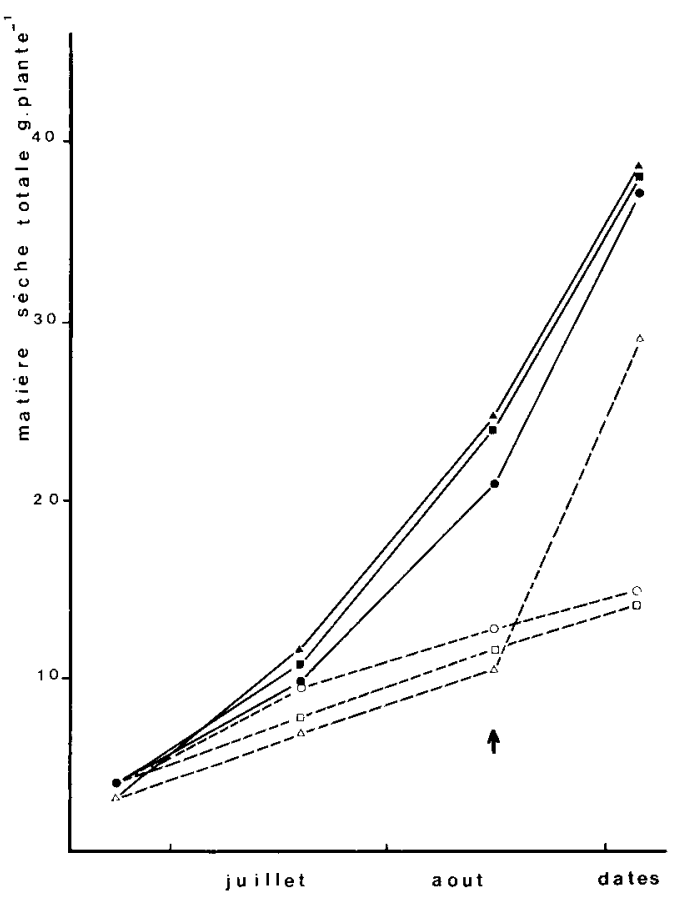

Figure 2

Evolution de la matière sèche totale (g.plante ${ }^{-1}$ ) des organes aériens. Même légende que pour la figure 1.

Total shoot dry matter (g.plant ${ }^{-1}$ ). Legend as for figure 1 .

\section{B. Photosynthèse et transpiration}

Le déficit hydrique, qui se traduit par une forte diminution du potentiel de l'eau dans les feuilles, augmente l'ensemble des résistances à la carboxylation, notamment la résistance stomatique (tabl. 3) et réduit la photosynthèse nette. La variété " Jiangedou » est particulièrement touchée : sa photosynthèse est le tiers de celle du témoin ; pour «Q.533 » et « Kingsoy», elle est réduite de moitié. 
TABLEAU 2

Fructification, production et composition des graines (moyenne de 10 plantes). Les pieds témoins sont dans des conditions d'évapotranspiration maximale depuis le semis. Les pieds traités ont subi un déficit hydrique important jusqu'au 15 aô̂t ; ils sont ensuite placés dans les mêmes conditions que les témoins.

Fruiting, seed production and composition (mean of ten plants). Standard plots were in maximum evapotranspiration conditions from seeding. Treated plots were submitted to water stress until mid-August; after, same conditions as standards.

\begin{tabular}{|c|c|c|c|c|c|c|}
\hline & \multicolumn{2}{|c|}{ Kingsoy } & \multicolumn{2}{|c|}{ Jiangedou } & \multicolumn{2}{|c|}{ Q.533 } \\
\hline & Témoin & Traité & Témoin & Traité & Témoin & Traité \\
\hline Nombre de gousses par plante & $31,7 \pm 1,3$ & $23,3 \pm 2,1$ & $27,5 \pm 2,4$ & $21,1 \pm 1,9$ & $86,2 \pm 15,3$ & $33,5 \pm 13,3$ \\
\hline Nombre de graines par plante & $64,9 \pm 3,3$ & $35,9 \pm 3,9$ & $51,9 \pm 5,2$ & $31,6 \pm 2,6$ & $105 \pm 21$ & $43,3 \pm 17,9$ \\
\hline Nombre de graines par gousse & 2,05 & 1,54 & 1,89 & 1,50 & 1,22 & 1,29 \\
\hline $\begin{array}{l}\text { Masse moyenne d'une graine } \\
\text { (mg) }\end{array}$ & $192 \pm 6,4$ & $192 \pm 3,5$ & $221 \pm 3$ & $204 \pm 12$ & $105 \pm 1$ & $115 \pm 8$ \\
\hline Masse des graines (g.plante ${ }^{-1}$ ) & $12,4 \pm 0,3$ & $6,9 \pm 0,7$ & $11,5 \pm 1,1$ & $6,4 \pm 0,6$ & $11,0 \pm 2,3$ & $4,9 \pm 2,1$ \\
\hline $\begin{array}{l}\text { Teneur en protéines des graines } \\
(\%)\end{array}$ & 44,2 & 44,0 & 45,9 & 46,2 & 45,6 & 44,9 \\
\hline $\begin{array}{l}\text { Teneur en huile des graines } \\
(\%)\end{array}$ & 21,0 & 20,8 & 19,2 & 17,9 & 17,1 & 17,7 \\
\hline
\end{tabular}

TABLEAU 3

Potentiel hydrique (MPa), résistance stomatique (s. $m^{-1}$ ) et photosynthèse ( $\mu m o l e ~ \mathrm{CO}_{2} \cdot m^{-2} . s^{-1}$ ) des feuilles. Les mesures sont effectuées en milieu de journée. Le 11 août correspond à la période de déficit hydrique; le 26 août, la contrainte hydrique est terminée depuis 10 jours.

Leaf water potentiel (MPa), stomatal resistance (s. $m^{-1}$ ) and photosynthesis ( $\mu$ mole $\left.\mathrm{CO}_{2} \cdot \mathrm{m}^{-2} \cdot \mathrm{s}^{-1}\right)$. Measurements were made around noon, during water stress (August 11) and 10 days after the end of water stress (August 26).

\begin{tabular}{|c|c|c|c|c|c|c|}
\hline & \multicolumn{2}{|c|}{ Kingsoy } & \multicolumn{2}{|c|}{ Jiangedou } & \multicolumn{2}{|c|}{ Q.533 } \\
\hline & Témoin & Traité & Témoin & Traité & Témoin & Traité \\
\hline \multicolumn{7}{|c|}{ Potentiel hydrique } \\
\hline $\begin{array}{l}11 \text { août } \\
\text { Résistan }\end{array}$ & $-0,87 \pm 0,16$ & $-2,0 \pm 0,3$ & $-1,0 \pm 0,09$ & $-2,0 \pm 0,03$ & $-0,88 \pm 0,20$ & $-1,2 \pm 0,02$ \\
\hline 11 août & $119+24$ & $605 \pm 113$ & $196 \pm 60$ & $2770 \pm 335$ & $210 \pm 21$ & $1294 \pm 414$ \\
\hline 26 août & $71 \pm 13$ & $147 \pm 27$ & $101 \pm 22$ & $109 \pm 10$ & $84 \pm 14$ & $149 \pm 32$ \\
\hline \multicolumn{7}{|c|}{ Photosynthèse } \\
\hline 11 août & $5,9 \pm 0,9$ & $3,4 \pm 1,2$ & $6,9 \pm 1,4$ & $2,3 \pm 0,7$ & $9,8 \pm 2,7$ & $9,8 \pm 1,2$ \\
\hline 26 août & $12,6 \pm 2,0$ & $11,1 \pm 0,9$ & $10,2 \pm 1,9$ & $9,1 \pm 1,0$ & $14,1 \pm 2,7$ & $10,7 \pm 1,2$ \\
\hline
\end{tabular}

La transpiration est mesurée au cours de journées caractéristiques de périodes bien ensoleillées, mais présentant différentes conditions de température et d'hygrométrie (fig. 3). Elle s'applique à l'unité de surface de la face inférieure de feuilles adultes bien ensoleillées. Ces mesures sont réalisées en cours d'aprèsmidi, après la « dépression de midi ». Elles mettent en évidence plusieurs faits :

- le comportement des plantes témoins diffère selon les cultivars : par forte demande évaporative, la transpiration est toujours modérée pour la feuille de " Jiangedou » et de «Q.533 », alors qu'elle est très élevée pour « Kingsoy ». En revanche, si la demande évaporative est moins importante, la régulation stomatique s'exerce peu et la consommation d'eau de "Q.533 » peut être très grande ; c'était le cas au début du mois de septembre ;

- pendant la période de déficit hydrique, la transpiration des plantes traitées, qui diminue fortement, traduit la sévérité de la contrainte subie ;

- à partir de la réhumidification du sol, la transpiration rejoint progressivement, en une quinzaine de jours environ, celle des plantes témoins, même lorsque celle-ci est très élevée, ce qui est alors le cas de «Q.533 ».

\section{Métabolisme carboné}

\section{La feuille}

Trois jours après l'incorporation du ${ }^{14} \mathrm{CO}_{2}$, la radioactivité foliaire est encore élevée et sa répartition entre les différentes catégories de composés organiques varie suivant les cultivars (tabl. 4). Ainsi, pour les plantes témoins, la part de radiocarbone incorporée dans les composés insolubles passe de 30 p. 100 pour « Kingsoy » à 42 p. 100 pour «Q.533 ». Mais ces proportions sont toujours plus élevées dans les feuilles en déficit hydrique : «Kingsoy », 39 p. 100 ; « Jiangedou », 40 p. 100 et «Q.533», 57 p. 100. Ceci est dû essentiellement à une augmentation du marquage des composés non protéiques : amidon, cellulose, lignine, etc... En revanche, la radioactivité de la fraction neutre soluble est plus faible. Dans cette dernière, les métabolites les plus marqués sont, dans l'ordre décroissant : le glucose, le fructose, le saccharose, le mélibiose, le raffinose, le stachyose... Pour ce qui est des acides organiques, ils contiennent entre 24 et 32 p. 100 du radiocarbone : les acides malique et citrique sont les plus marqués ; viennent ensuite les acides fumarique, malonique, succinique... 


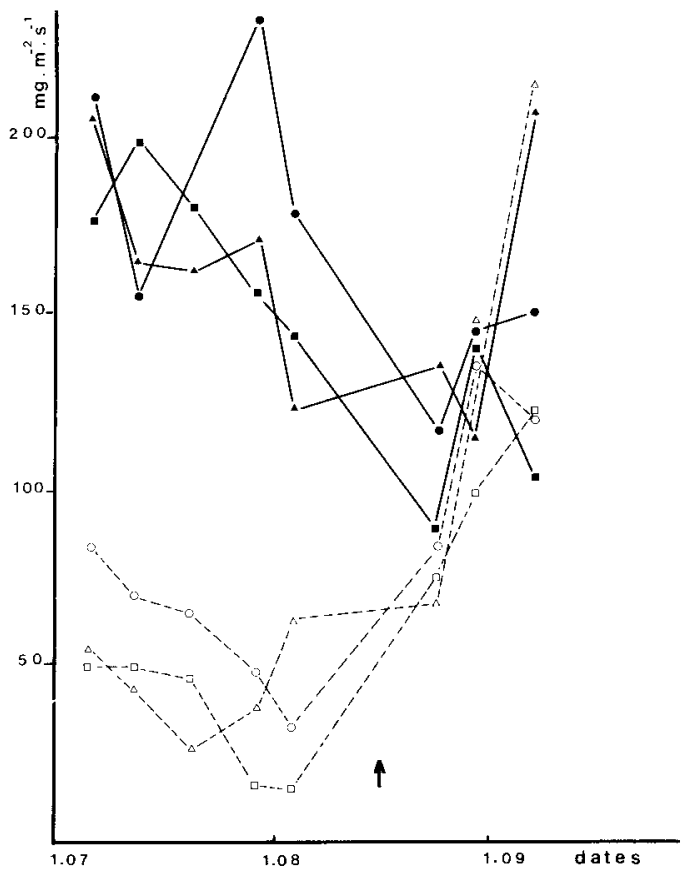

Figure 3

Transpiration ( $m g$ d'eau. $\left.m^{-2} \cdot s^{-1}\right)$ de la face inférieure de feuilles bien ensoleillées. Même légende que pour la figure 1. La température $\left({ }^{\circ} \mathrm{C}\right)$, l'humidité relative $(\%)$ et l'éclairement $\left(\mu E \cdot s^{-1} \cdot m^{-2}\right)$ moyens sont respectivement : 30, 50, 1650 (7-22 juillet); 34, 43, 1800 (30 juillet) ; 28, 58, $1550 \quad(25-31$ août $)$ et $29,38,1650$ (10 septembre).

Transpiration (mg water. $\mathrm{m}^{-2} \cdot \mathrm{s}^{-1}$ ) of well illuminated leaves (lower surface). Legend for figure 1 . Temperature $\left({ }^{\circ} \mathrm{C}\right)$, relative humidity (\%), light intensity $\left(\mu \mathrm{E} . \mathrm{s}^{-1} \cdot \mathrm{m}^{-2}\right)$ respectively : 30, 50, 1650 (July 7-22) ; 34, 43, 1800 (July 30) ; 28, 58, 1550 (August 25-31) ; 29, 38, 1650 (September 10).

Par rapport au ${ }^{14} \mathrm{CO}_{2}$, l'incorporation de glycolate$1{ }^{14} \mathrm{C}$ se caractérise par un marquage plus important des composés azotés solubles et insolubles (tabl. 4). L'étude détaillée des autoradiogrammes montre que le radiocarbone se trouve surtout dans les molécules de glycine et sérine. Tandis que l'assimilation de ${ }^{14} \mathrm{CO}_{2}$ permet de suivre le devenir de l'ensemble du carbone fixé, l'incorporation de glycolate-1-14 $\mathrm{C}$ entraîne un marquage préférentiel des métabolites de la voie photorespiratoire à laquelle appartiennent précisément la glycine et la sérine. Une partie de ces $\mathbf{2}$ acides aminés est ensuite prélevée pour les synthèses protéiques (CAlmés \& Viala, 1981). Comme dans le cas du ${ }^{14} \mathrm{CO}_{2}$, l'incorporation de radiocarbone dans la fraction protéique insoluble est moindre avec les plantes traitées qu'avec les témoins.

\section{Migrations vers les gousses}

Lors de la $1^{\text {re }}$ expérimentation avec du ${ }^{14} \mathrm{CO}_{2}$, les gousses des plantes irriguées sont peu développées, les témoins de la variété «Q.533 » sont même en pleine floraison. Au contraire, les plantes en déficit hydrique se trouvent à un stade physiologique plus avancé ; la masse de leurs gousses comparée à celle de la feuille est nettement supérieure à celle des témoins. Il en résulte un effet «puits » plus grand et la répartition $\mathrm{du}$ radiocarbone entre les organes est difficilement comparable.

Dix jours après la fin du déficit hydrique, une nouvelle incorporation de ${ }^{14} \mathrm{CO}_{2}$ est effectuée. La figure 4 traduit, pour les 3 variétés, la distribution du radiocarbone entre la feuille et les fructifications. Les différences entre les 3 plantes témoins sont assez faibles; les migrations vers les gousses sont un peu moins importantes pour «Q.533 » que pour « Jiangedou » et « Kingsoy ». Les plantes soumises antérieurement à une longue période de déficit hydrique présentent des migrations comparables à celles des témoins en ce qui concerne « Kingsoy » et " Jiangedou ». En revanche, «Q.533 », dont les gousses sont désormais bien développées, se caractérise par un apport très faible de radiocarbone vers les fructifications : 4 fois moindre que pour le témoin. Dans ce cas, la nutrition carbonée des graines subit encore les répercussions d'une sécheresse qui a cessé depuis $10 \mathrm{j}$.

\section{TABLEAU 4}

Répartition (\%) de la radioactivité foliaire $72 \mathrm{~h}$ après incorporation de ${ }^{14} \mathrm{CO}_{2}$ ou de glycolate ${ }^{14} \mathrm{C}$ pendant la période de déficit hydrique (11 août).

Distribution of label (\%) in the leaf, $72 \mathrm{~h}$ after ${ }^{14} \mathrm{CO}_{2}$ or ${ }_{1-}^{14} \mathrm{C}$-glycolate incorporation during water stress (August 1I).

\begin{tabular}{|c|c|c|c|c|c|c|}
\hline & \multicolumn{2}{|c|}{ Kingsoy } & \multicolumn{2}{|c|}{ Jiangedou } & \multicolumn{2}{|c|}{ Q.533 } \\
\hline & Témoin & Traité & Témoin & Traité & Témoin & Traité \\
\hline \multicolumn{7}{|l|}{${ }^{14} \mathrm{CO}_{2}$} \\
\hline \multicolumn{7}{|l|}{ Composés solubles } \\
\hline - neutres & 32,9 & 24,3 & 36,0 & 25,4 & 26,2 & 16,1 \\
\hline - acides organiques & 32,1 & 32,0 & 24,0 & 30,9 & 28,1 & 23,6 \\
\hline — acides aminés & 4,6 & 4,5 & 4,8 & 3,6 & 3,2 & 3,0 \\
\hline \multicolumn{7}{|l|}{ Composés insolubles } \\
\hline - protéiques & 18,7 & 20,3 & 19,6 & 18,9 & 11,9 & 12,0 \\
\hline - non protéiques & 11,7 & 18,9 & 15,6 & 21,3 & 30,6 & 45,3 \\
\hline \multicolumn{7}{|l|}{ Glycolate-1 ${ }^{14} \mathrm{C}$} \\
\hline \multicolumn{7}{|l|}{ Composés solubles } \\
\hline - neutres & 20,5 & 20,9 & 30,1 & 21,1 & 23,0 & 24,5 \\
\hline - acides organiques & 31,9 & 30,5 & 26,0 & 27,8 & 31,3 & 19,8 \\
\hline - acides aminés & 6,7 & 6,9 & 6,0 & 8,0 & 4,9 & 3,4 \\
\hline \multicolumn{7}{|l|}{ Composés insolubles } \\
\hline - protéiques & 24,9 & 20,6 & 19,7 & 20,4 & 20,2 & 13,7 \\
\hline - non protéiques & 16,0 & 21,1 & 18,2 & 22,7 & 20,6 & 38,6 \\
\hline
\end{tabular}




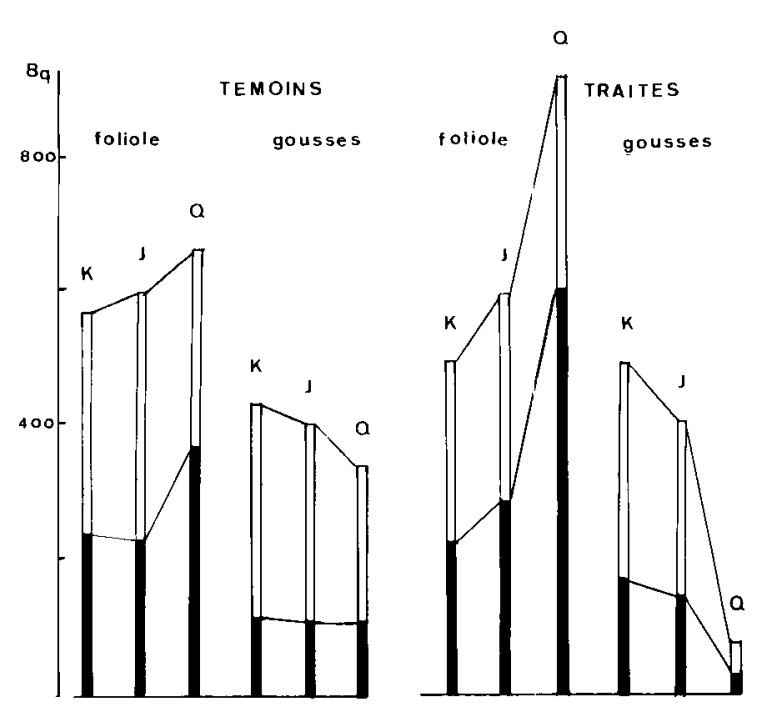

Figure 4

Répartition de la radioactivité entre la feuille et les gousses d'un même étage, $72 \mathrm{~h}$ après l'incorporation de ${ }^{14} \mathrm{CO}_{2}$. Les résultats sont exprimés en $B q$ pour un total de 1000 dans l'ensemble feuille et gousses. L'expérimentation est effectuée le 26 août, 10 jours après la fin du stress hydrique. $K$ : Kingsoy; $J:$ Jiangedou; $Q: 533$; $\square$, soluble; $\mathbf{\square}$, insoluble.

Distribution of label between leaf and pods of same level, $72 \mathrm{~h}$ after ${ }^{14} \mathrm{CO}_{2}$ incorporation. Data in $\mathrm{Bq}$ per 1000 for leaf and pods together. Values correspond to August 26, 10 days after end of drought : K, Kingsoy ; J, Jiangedou ; $Q, Q .533$; $\square$, soluble ; $\mathbf{\square}$, insoluble.

\section{Métabolisme azoté}

L'évolution, au cours du développement, de la quantité d'azote présente dans les organes aériens est illustrée sur la figure 5. Aucun engrais azoté n'ayant été apporté, l'alimentation en cet élément dépend surtout de la fixation symbiotique. Or Bouniols et al. (1982), OBATON et al. (1982), ont montré que celle-ci est extrêmement réduite chez les plantes soumises à la sécheresse. Lorsque le sol est réhumidifié, la nutrition azotée reprend modérément chez «Kingsoy » et « Jiangedou » et très activement chez «Q.533 " où elle correspond à une très forte reprise de la croissance.

La baisse de la teneur en azote due au déficit hydrique se répercute sur la répartition du radiocarbone incorporé. La comparaison du marquage de la fraction protéique par rapport à celui de l'ensemble de la fraction insoluble, établie à l'aide des données du tableau 4, montre que les protéines sont plus marquées dans les témoins que dans les pieds traités, aussi bien dans la feuille que dans la gousse : le déficit hydrique ralentit la protéogenèse.

Par rapport aux témoins, les feuilles en déficit hydrique présentent toujours des teneurs en acides aminés libres plus élevées : respectivement 90 et 48 mmoles/g de matière sèche pour «Kingsoy », 76 et 62 pour «Jiangedou », 57 et 50 pour «Q.533». En ce qui concerne l'importance relative de chaque acide, on observe peu de différences pour la variété «Q.533 ». En revanche, pour « Kingsoy » et « Jiangedou », la sécheresse entraîne un déficit en acide $\gamma$-aminobutyrique et alanine et un excès de proline et d'asparagine.

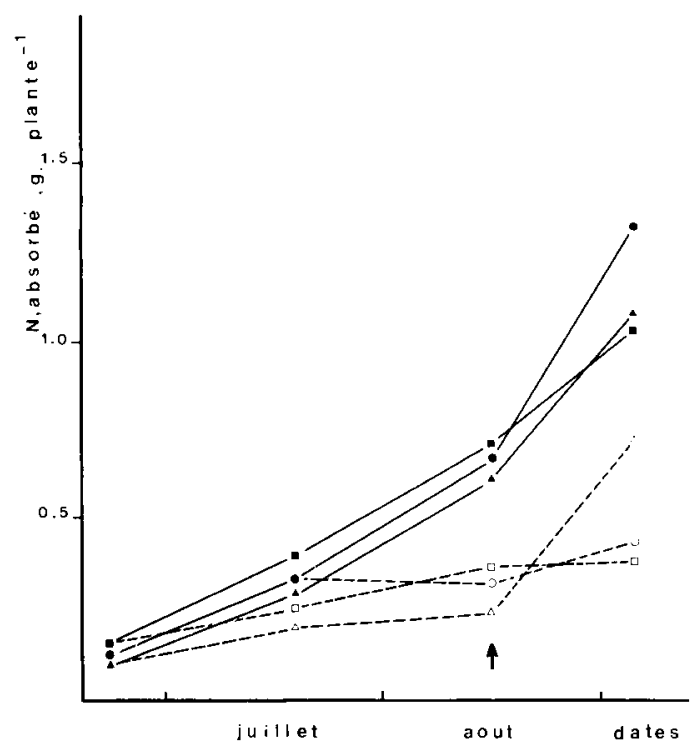

Figure 5

Quantité d'azote (g.plante-1) contenue dans les parties aériennes. Même légende que pour la figure 1 .

Total nitrogen content of shoots (g.plant $\left.{ }^{-1}\right)$. Legend as for figure 1 .

Les acides aminés les plus radioactifs, $72 \mathrm{~h}$ après l'incorporation de ${ }^{14} \mathrm{CO}_{2}$, sont les acides $\gamma$-aminobutyrique et glutamique, l'asparagine et l'alanine : ils contiennent de 70 à 80 p. 100 de radiocarbone alors qu'ils ne représentent que 56 à 76 p. 100 de l'ensemble des acides aminés libres.

Dix jours après la fin du déficit hydrique, à l'époque du grossissement des graines, «Kingsoy » est la variété qui incorpore la plus grande quantité de radiocarbone dans ses protéines (fig. 6) : 24 p. 100 pour le témoin et 22 pour le pied traité, si l'on considère l'ensemble foliole + gousses; l'effet de la période de sécheresse s'est considérablement atténué. "Jiangedou » en incorpore respectivement 20 et 17 p. 100 : les conséquences du déficit hydrique persistent encore. «Q.533 » est la variété qui intègre le moins de radiocarbone dans les protéines et le manque d'eau rend cette intégration encore plus faible (6 p. 100 au lieu de 10 pour le témoin); de plus, les migrations vers les gousses sont profondément ralenties en particulier

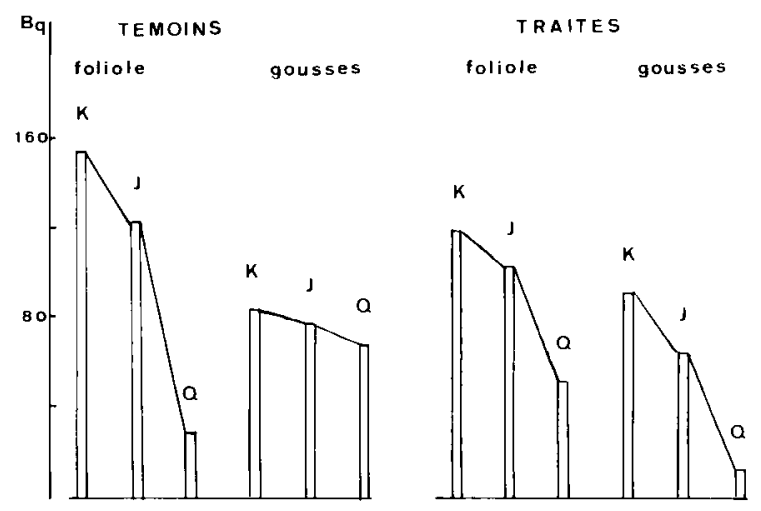

Figure 6

Fraction de la radioactivité incorporée dans les protéines. Même légende que pour la figure 4.

Label fraction incorporated into protein. Legend as for figure 4. 
pour le pied traité où le marquage des protéines des fructifications est près de 8 fois plus faible que celui de la variété « Kingsoy».

\section{DISCUSSION}

Le déficit hydrique du mois de juillet et de la $1^{\text {re }}$ quinzaine d'août 1983 se traduit par une diminution des processus photosynthétiques, accompagnée d'un déséquilibre entre les métabolismes carboné et azoté. D'après nos résultats, le carbone récemment fixé est incorporé en plus grande proportion dans les composés insolubles non protéiques: l'orientation vers la protéogenèse est particulièrement déficitaire pour «Q.533 ", aussi bien après incorporation de ${ }^{14} \mathrm{CO}_{2}$ que de glycolate- $1-{ }^{14} \mathrm{C}$.

La voie photorespiratoire, que ce dernier précurseur permet de suivre, est exaltée par la sécheresse (BENGOUA, 1982). Comme elle comporte une orientation plus grande du radiocarbone vers la synthèse protéique, spécialement sous forme de glycine et de sérine, on pourrait s'attendre à ce que les pieds en déficit hydrique incorporent dans les protéines davantage de radiocarbone que les témoins bien irrigués. En fait, il n'en est rien : on ne note aucune différence significative lors de la maturité des graines.

Bien que l'activité des nodosités (PATTERSON et al., 1979 ; CHEN \& SUNG, 1983 ; SUNG \& CHEN, 1983) soit fortement diminuée par la sécheresse, les acides aminés libres sont abondants dans les feuilles : ils contribuent au maintien d'une pression osmotique élevée (FUKUTOKU \& YAMADA, 1982 ; HANDA et al., 1983). L'activité moindre du métabolisme azoté n'affecte pas la synthèse des acides aminés, mais leur assemblage en chaînes polypeptidiques. Ceci rejoint un fait souvent constaté, la disparition des polyribosomes lors des déficits hydriques (BRADFORD \& HSIAO, 1982).
Dix jours après la fin du déficit hydrique, la protéogenèse n'a pas encore repris son importance normale ; le marquage de la fraction protéique reste déficitaire. Ce déficit, important dans les feuilles en particulier pour «Q.533», est beaucoup plus limité dans les graines. A maturité, la teneur en protéines des graines sera sensiblement la même pour les témoins et les traités des 3 variétés. En accord avec les travaux de DovE (1982), le déficit hydrique n'a eu de répercussions que sur le nombre et la masse des graines produites. Toutefois, dans d'autres conditions de contrainte hydrique, en particulier si elle est plus tardive, la teneur en protéines des graines peut être modifiée (BoUNIOLS et al., 1982).

Sur le plan agronomique, il est assez remarquable de constater que les 3 cultivars ont, pendant la phase de sécheresse, des comportements voisins. «Jiangedou "), qui consomme moins d'eau lorsque celle-ci est disponible, ne manifeste pas une meilleure tolérance au déficit hydrique que «Kingsoy ». Ce dernier, dont le métabolisme azoté est bien orienté vers les gousses, est actuellement l'une des variétés à la fois les plus productives et les plus robustes en conditions sèches.

Le comportement de « Q.533 » est plus original : sa capacité de reprise de croissance est remarquable. Par contre, la fructification de ce cultivar est plus sensible que celle des 2 autres ; peut-être la contrainte a-t-elle été levée un peu tard dans cette expérience pour que la fructification puisse bénéficier de la reprise de croissance : ce point fera l'objet d'études ultérieures. Cette excellente faculté de reprise n'est sans doute pas très facile à utiliser car elle est insuffisamment orientée vers les graines notamment quant au métabolisme azoté. Toutefois, «Q.533» pourrait présenter un intérêt à titre de géniteur pour tenter d'améliorer la tolérance de l'espèce aux aléas de sécheresses susceptibles de survenir à divers stades du développement.

Reçu le $1^{\text {er }}$ juin 1984 Accepté le 5 octobre 1984.

\section{RÉFÉRENCES BIBLIOGRAPHIQUES}

Baldocchi D. D., Verma S. B., Rosenberg N. J., Blad B. L., Garay A., Specht J. E., 1983. Influence of water stress on the diurnal exchange of mass and energy between the atmosphere and a soybean canopy. Agron. J., 75, 543-548.

Bengoua A., 1982. Echanges gazeux et métabolisme photorespiratoire du soja : influence de l'ombre et de la sécheresse. Thèse Doct. Ing., Toulouse, U.P.S., 101 p.

Blanchet R., Gelfi N., Merrien A., Puech J., 1982. Studies on some characters of water consumption and water use efficiency on some soybean varieties. Soybean Sci., 1, 17-32.

Bouniols A., Puech J., Mondies M., 1982. Influence d'un déficit hydrique appliqué durant la fructification sur la production du soja. Bull. Gr. Fr. Humid. Neutron, 11, 39-56.

Bradford K. J., Hsiao T. C., 1982. Physiological responses to moderate water stress. In Encyclopedia of Plant Physiology, New series, Vol. 12 B, Physiological Plant Ecology II. Water relations and carbon assimilation, Lange O. L., Nobel P. S., Osmond C. B. and Ziegler H. eds. Springer Verlag, Heidelberg, New York, 263324.

Calmés J., Latché J. C., Cavalié G., 1977. Métabolisme du $1{ }^{14} \mathrm{C}$ glycolate et biosynthèse de la sérine chez le soja. Plant Sci. Lett., 9 , 239-246.

Calmés J., Viala G., 1981. Protéogenèse dans les graines de soja : participation de la glycine et de la sérine formées dans les feuilles au cours du métabolisme photorespiratoire. C. R. Acad. Sci., Paris, série III, 292, 353-355.

Chen C. L., Sung J. M., 1983. Effect of water stress on the reduction of nitrate and nitrite by soybean nodules. Plant Physiol., 73, 1065-1066.

Dove L., 1982. Protein formation in developing soybean (Glycine $\max$ (L.) Merr.) seeds and its resistance to water stress. New Physiol., 91, 177-182.

Finn G. A., Brun W. A., 1980. Water stress effects on $\mathrm{CO}_{2}$ assimilation, photosynthate partitioning, stomatal resistance and nodule activity in soybean. Crop Sci., 20, 431-434.

Fukutoku Y., Yamada Y., 1982. Accumulation of carbohydrates and proline in water-stressed soybean (Glycine max). Soil Sci. Plant Nutr., 28, 147-151.

Handa S., Bressan R. A., Handa A. K., Carpita N. C., Hasegawa P. M., 1983. Solutes contributing to osmotic adjustment in cultured plant cells adapted to water stress. Plant Physiol., 73, 834-843.

Hsiao T. C., 1973. Plant responses to water stress. Annu. Rev. Plant Physiol., 24, 519-570.

Huck M. G., Ishihara K., Peterson C. M., Ushijama T., 1983. Soybean adaptation to water stress at selected stages of growth. Plant Physiol., 73, 422-427. 
Maertens C., Blanchet R., 1981. Influence des caractères hydriques du milieu racinaire et aérien sur le potentiel de l'eau dans les feuilles de quelques types variétaux de soja et confrontation à leur comportement agronomique. Agronomie, 1, 199-206.

Obaton M., Miquel M., Robin P., Conejero G., Domenach A. M., Bardin R., 1982. Influence du déficit hydrique sur l'activité nitrate réductase et nitrogénase chez le soja. (Glycine max (L.) Merr., cv "Hodgson »). C. R. Acad. Sci., Paris, série III, 294, 1007-1012.

Paleg L. G., Aspinall D., 1981. The physiology and biochemistry of drought resistance in plants. Academic Press, Sydney, New York, London, Toronto, San Francisco, 492 p.

Patterson R. P., Raper Jr C. D., Gross H. D., 1979. Growth and specific nodule activity of soybean during application and recovery of a leaf moisture stress. Plant Physiol., 64, 551-556.

Planchon C., Vignes D., 1978. Etude de la transpiration et de la régulation stomatique de deux variétés de soja (Glycine max (L.) Merrill) : conséquences relatives à la création de types à exigences en eau réduites. Ann. Amélior. Plant., 28, 149-155.
Pognonec J. C., 1983. Contribution à la recherche d'un critère de sélection pour l'adaptation à la sécheresse du soja. Thèse Univ. Sciences et Techniques Languedoc, 207 p.

Sammons D. J., Peters D. B., Hymowitz T., 1978. Screening soybeans for drought resistance. I. Growth chamber procedure. Crop Sci., 18, 1050-1055.

Sammons D. J., Peters D. B., Hymowitz T., 1979. Screening soybeans for drought resistance. II. Drought procedure. Crop Sci., 19, 719-722.

Shimshi D., 1969. A rapid field method of measuring photosynthesis with labelled carbon dioxide. Isr. J. Bot., 20, 381-401.

Sung J. M., Chen C. L., 1983. The effect of water stress on enzymes of ammonia assimilation on cytosol of soybean nodules. $J$. Agric. Assoc. China, 121, 20-34.

Vidal A., Arnaudo D., Arnoux M., 1981. La résistance à la sécheresse du soja. Agronomie, 1, 295-313. 